\title{
CCD SDSS gr Photometry of Poorly Studied Star Clusters in the Large Magellanic Cloud
}

\author{
Andrés E. Piatti ${ }^{1,2}$ \\ ${ }^{1}$ Observatorio Astronómico, Universidad Nacional de Córdoba, Laprida 854, 5000 Córdoba, Argentina \\ ${ }^{2}$ Consejo Nacional de Investigaciones Científicas y Técnicas, Avenida Rivadavia 1917, C1033AAJ Buenos Aires, Argentina
}

Correspondence should be addressed to Andrés E. Piatti; andres@oac.uncor.edu

Received 19 November 2013; Accepted 18 March 2014; Published 11 May 2014

Academic Editors: M. Marconi and L. Pulone

Copyright (C) 2014 Andrés E. Piatti. This is an open access article distributed under the Creative Commons Attribution License, which permits unrestricted use, distribution, and reproduction in any medium, provided the original work is properly cited.

\begin{abstract}
We present for the first time CCD SDSS gr photometry, obtained at the Gemini South telescope with the GMOS attached, of stars in the field of the poorly studied star clusters NGC1768, HS 85, SL 676, NGC2107, NGC2190, and SL 866, which are distributed in the main body of the Large Magellanic Cloud. We applied a subtraction procedure to statistically clean the cluster CMDs from field star contamination. In order to disentangle cluster features from those belonging to their surrounding fields, we applied a subtraction procedure which makes use of variable cells to reproduce the field star color-magnitude diagrams (CMDs) as closely as possible. We then traced their stellar density radial profiles from star counts performed over the cleaned field stars dataset and derived their radii. Using the cleaned cluster CMDs, we estimated ages and metallicities from matching theoretical isochrones computed for the SDSS system. The studied star clusters have ages from $0.1 \mathrm{up}$ to $2.0 \mathrm{Gyr}$ and are of slightly metal-poor metal content ([Fe/ $\mathrm{H}] \approx-0.4 \mathrm{dex})$.
\end{abstract}

\section{Introduction}

The Large Magellanic Cloud (LMC) harbors more than two thousand catalogued ordinary star clusters [1]. Although they are prime indicators of the chemical evolution and the star formation history of the galaxy, only a very small percentage has been well studied $[2,3]$. In this sense, detailed investigations of even a handful of clusters represent a significant improvement of our knowledge of the chemical enrichment history of this galaxy.

We have been intensively involved in a long-term project aimed at obtaining ages and metallicities of LMC clusters, as well as addressing other important related issues. For instance, we have discovered a new giant branch clump structure [4], studied the infamous cluster age-gap [5], searched for age and metallicity gradients [6], derived ages and metallicities for some 81 LMC clusters [7, 8], and investigated in detail the LMC field and cluster age-metallicity relationships [9], among others. We continue here our previous work on LMC clusters by presenting results for the six mostly unstudied clusters (NGC 1768, HS 85, SL 676, NGC 2107, NGC 2190, and SL 866) with the aim of adding them to our growing sample of well-studied LMC clusters that will allow us to assemble a much more comprehensive database to study the formation and evolution of LMC clusters and their parent galaxy.

The paper is organized as follows. The next section describes the collected observations and the data reduction. Section 3 deals with the observed color-magnitude diagrams (CMDs) and the procedure of disentangling cluster from field star features. We focus also on the estimation of the cluster structural parameters. The cluster fundamental parameters are derived in Section 4, while the analysis and discussion of the results are presented in Section 5. Our main findings are summarized in Section 6.

\section{Data Handling}

Based on data obtained from the Gemini Science Archive, we collected CCD SDSS gr [11] images centered on 6 LMC clusters (GS-2010B-Q-74, PI: Pessev) along with observations of standard fields and calibration frames (zero, sky-flat, and dome-flat). The data were obtained at the Gemini South telescope with the Gemini multiobject spectrograph (GMOS) 
TABLE 1: Observation log of selected LMC clusters.

\begin{tabular}{|c|c|c|c|c|c|c|}
\hline Star cluster & $\alpha_{2000}(\mathrm{~h} \mathrm{~m} \mathrm{~s})$ & $\delta_{2000}\left({ }^{\circ \prime \prime \prime}\right)$ & Filter & Exposures (times $\times \mathrm{sec}$ ) & Airmass & Seeing $^{\prime \prime}$ \\
\hline \multirow{2}{*}{ NGC 1768} & \multirow{2}{*}{045702} & \multirow{2}{*}{-681456} & $g$ & $2 \times 30$ & 1.283 & 1.2 \\
\hline & & & $r$ & $2 \times 15$ & 1.281 & 1.1 \\
\hline \multirow{2}{*}{ HS 85} & \multirow{2}{*}{050051} & \multirow{2}{*}{-674814} & $g$ & $2 \times 30$ & 1.308 & 0.9 \\
\hline & & & $r$ & $2 \times 15$ & 1.306 & 0.9 \\
\hline \multirow{2}{*}{ SL 676} & \multirow{2}{*}{054309} & \multirow{2}{*}{-703416} & $g$ & $2 \times 30$ & 1.321 & 0.7 \\
\hline & & & $r$ & $2 \times 15$ & 1.322 & 0.6 \\
\hline \multirow{2}{*}{ NGC 2107} & \multirow{2}{*}{054313} & \multirow{2}{*}{-703823} & $g$ & $2 \times 30$ & 1.321 & 0.7 \\
\hline & & & $r$ & $2 \times 15$ & 1.322 & 0.6 \\
\hline \multirow{2}{*}{ NGC 2190} & \multirow{2}{*}{060102} & \multirow{2}{*}{-744333} & $g$ & $2 \times 30$ & 1.509 & 1.2 \\
\hline & & & $r$ & $2 \times 15$ & 1.503 & 1.1 \\
\hline \multirow{2}{*}{ SL 866} & \multirow{2}{*}{061432} & \multirow{2}{*}{-655857} & $g$ & $2 \times 30$ & 1.407 & 1.1 \\
\hline & & & $r$ & $2 \times 15$ & 1.402 & 1.0 \\
\hline
\end{tabular}

attached (scale $=0.146 \mathrm{arcsec} / \mathrm{pixel}$ ). The log of the observations is presented in Table 1, where the main astrometric, photometric, and observational information is summarized. Nine Gemini Observatory standard fields were observed along the 5 cluster observing nights as baseline observations, for which 2 exposures of $5 \mathrm{~s}$ per filter and airmass in the range $\sim 1.0-2.0$ were obtained.

The data reduction followed the procedures documented in the Gemini Observatory webpage (http://www.gemini. edu) and utilized the GEMINI/GMOs package in IRAF (IRAF is distributed by the National Optical Astronomy Observatories, which is operated by the Association of Universities for Research in Astronomy, Inc., under contract with the National Science Foundation). We performed overscan, trimming, and bias subtraction, flattened all data images, and so forth, once the calibration frames (zeros and flats) were properly combined. The final field of view of the images resulted to be $\sim 5.6^{\prime} \times 5.6^{\prime}$.

Around 30-50 independent magnitude measures of standard stars were derived per filter using the АРРнот task within IRAF, in order to secure the transformation from the instrumental to the SDSS gr standard system. Standard stars were distributed over an area similar to that of the GMOS array, so that we measured magnitudes of standard stars in each of the three chips. The relationships between instrumental and standard magnitudes were obtained by fitting the following equations:

$$
\begin{gathered}
g=g_{1}+g_{\text {std }}+g_{2} \times X_{g}+g_{3} \times(g-r)_{\text {std }} \\
r=r_{1}+r_{\text {std }}+r_{2} \times X_{r}+r_{3} \times(g-r)_{\text {std }},
\end{gathered}
$$

where $g_{i}$ and $r_{i}(i=1,2,3)$ are the fitted coefficients and $X$ represents the effective airmass. We solved the transformation equations for the three chips with the FITPARAMs task in IRAF, simultaneously with the rms errors from the transformation to the standard system being 0.015 mag for $g$ and 0.023 for $r$, respectively, indicating an excellent match to the standard system.

The stellar photometry was performed using the starfinding and point-spread-function (PSF) fitting routines in the DAOРнот/ALLSTAR suite of programs [12]. For each frame, a quadratically varying PSF was derived by fitting $\sim 60$ stars, once the neighbors were eliminated using a preliminary PSF derived from the brightest, least contaminated 20-30 stars. Both groups of PSF stars were interactively selected. We then used the ALLSTAR program to apply the resulting PSF to the identified stellar objects and to create a subtracted image which was used to find and measure magnitudes of additional fainter stars. This procedure was repeated three times for each frame. Finally, we computed aperture corrections from the comparison of PSF and aperture magnitudes by using the neighbor-subtracted PSF star sample. After deriving the photometry for all detected objects in each filter, a cut was made on the basis of the parameters returned by DАОРнот. Only objects with $\chi<2$, photometric error less than $2 \sigma$ above the mean error at a given magnitude, and $\mid$ SHARP $\mid<0.5$ were kept in each filter (typically discarding about $10 \%$ of the objects), and then the remaining objects in the $g$ and $r$ lists were matched with a tolerance of 1 pixel and raw photometry obtained.

We combined all the independent instrumental magnitudes using the stand-alone DAOMATCH and DAOMASTER programs, kindly provided by Peter Stetson. As a result, we produced one dataset per cluster containing the $x$ and $y$ coordinates for each star and two $(g, g-r)$ pairs. The gathered photometric information was standardized using (1). We finally averaged standard magnitudes and colors of stars measured twice. The resulting standardized photometric tables list successively a running number per star, the $x$ and $y$ coordinates, the averaged $g$ magnitudes, the observational errors $\sigma(g)$, the $g-r$ colors, the observational errors $\sigma(g-r)$, and the number of observations. We adopted the photometric errors provided by ALLSTAR (program kindly provided by P.B. Stetson) for stars with only one measure. Tables 2, 4 to 8 (available online in Supplementary Material at http://dx.doi.org/10.1155/2014/398182) provide this information for NGC 1768, HS 85, SL 676, NGC 2107, NGC 2190, and SL 866, respectively. Only a portion of Table 2 is shown here for guidance regarding its form and content. The whole content of Tables 2, 4-8 is provided as Supplementary Tables. 
TABLE 2: CCD gr data of stars in the field of NGC 1768.

\begin{tabular}{lccccccc}
\hline Star & $x(\mathrm{pixel})$ & $y(\mathrm{pixel})$ & $g(\mathrm{mag})$ & $\sigma(g)(\mathrm{mag})$ & $g-r(\mathrm{mag})$ & $\sigma(g-r)(\mathrm{mag})$ \\
\hline- & - & - & - & - & - & - & - \\
11 & 1493.480 & 1187.271 & 16.663 & 0.002 & -0.197 & 0.004 & 2 \\
12 & 1685.743 & 1275.260 & 16.605 & 0.002 & -0.226 & 0.004 & 0.003 \\
13 & 1874.385 & 611.421 & 16.661 & 0.002 & -608 & 2 \\
- & - & - & - & - & - & - \\
\hline
\end{tabular}

\section{Analysis of the Color-Magnitude Diagrams}

In order to obtain extracted CMDs where the fiducial features of the clusters can be clearly seen, we (i) cleaned the cluster CMDs from the field star contamination by using field stars placed beyond the cluster regions, (ii) determined the cluster geometrical centers, and (iii) traced the cluster radial profiles in order to determine the cluster extents.

As for cleaning the cluster CMDs from the field star contamination, we used the method developed by Piatti and Bica [13], which is designed to statistically reproduce the respective field star CMD and then to subtract it from the observed cluster CMD. The method is based on the fact that some parts of the field star CMD are more populated than others so that counting the number of stars within boxes of a fixed size becomes in a less profitable task. In general, bigger boxes are required to satisfactorily reproduce CMD regions with a small number of field stars, while smaller boxes are necessary in populous CMD regions. For instance, relatively bright field red giants with small photometric errors can be subtracted only if large enough boxes are used and, therefore, a cluster CMD without such a spurious red giant features can be built. Piatti and Bica proposed to use variable boxes in the field star CMDs. Magnitude and color box sizes are allowed to vary separately and fixed in such a way that they result in bigger CMD regions with a small number of stars, and vice versa. The boxes are placed and designed by taking into account the stellar density in the field star CMD, while the field stars are eliminated by looking for one star-the closest one in terms of magnitude and color-in the cluster CMD for each star identified in the field CMD. For our purposes, the field star CMDs were built using stars located typically beyond 700 pixels from the cluster centers. The bottom-right panel of Figures 1, 2, 3, 4, 5, and 6 shows the resulting boxes in the field CMD.

The coordinates of the cluster centers and their estimated uncertainties were determined by fitting Gaussian distributions to the star counts in the $x$ and $y$ directions for each cluster. These projected stellar densities were built using intervals of 40 pixel-wide, although we checked that using spatial bins from 20 to 60 pixels does not result in significant changes in the derived centers. We made use of the NGAUSSFIT routine in the STSDAS/IRAF package, which was executed from entering initial guesses for the single Gaussian's parameters, namely, a fixed constant-in our case equal to zero-which represents the corresponding background levels (i.e., stellar field densities assumed to be uniform), the linear terms to zero, the centers of the Gaussians, their amplitudes, and their full width at half-maximum (FWHM). We iterated the fitting procedure on average once, after eliminating a couple of discrepant points. Cluster centers were finally determined with a typical standard deviation of \pm 10 pixels $\left(\sim 1.5^{\prime \prime}\right)$ in all cases.

The cluster radial profiles were then obtained by first counting the number of stars in adjacent boxes of $20 \times$ 20 pixels covering the whole field of each cluster. Thus, at any distance $r$ from the cluster center, we computed the mean stellar density using the equation:

$$
\frac{\left(n_{r+10}-n_{r-10}\right)}{\left(m_{r+10}-m_{r-10}\right)},
$$

where $n_{j}$ and $m_{j}$ represent the sum of the number of stars counted in boxes closer than $j$ to the cluster centre and the number of boxes found inside $j$, respectively. Note that (2) provides us with the mean stellar density at a distance $j$ even though complete circles cannot be traced at that distance. This is an important consideration since having a stellar density profile, which extends far away from the cluster center, allows us to estimate the background level more precisely. Such profiles were in turn useful to derive the cluster radii, defined as the distance from the cluster center where the stellar density profile intersects the background level, as well as measuring the FWHM of the cluster density profiles, which play a significant role-from a stellar content point of view-in the construction of the cluster CMDs. When choosing the size of the rings, we preferred 20 pixels which allows us to statistically sample the stellar spatial distribution as well as avoiding spurious effects mainly caused by the presence of localized groups, rows, or columns of stars. Nevertheless, we traced the cluster radial profiles using rings with different sizes around 20 pixels-wide in order to estimate the uncertainties in the resulting radial profiles. Typically, the uncertainties vary from the center outwards with a $\mathrm{S} / \mathrm{N}$ ratio between 8 and 33 and the average being 14. The resulting density profiles are shown in the upper-right panel of Figures 1-6. We fitted a King [14] model to these stellar density profiles using the expression:

$$
\frac{N}{N_{o}}=\left(\frac{1}{\sqrt{1+\left(r / r_{c}\right)^{2}}}-\frac{1}{\sqrt{1+\left(r_{t} / r_{c}\right)^{2}}}\right)^{2}+b k g,
$$

where $N_{o}$ is the central stellar density and $r_{c}$ and $r_{t}$ are the core and tidal radii, respectively. $b \mathrm{~kg}$ represents the background level. $r_{c}$ and $r_{t}$ were estimated with a typical precision of 10 and 100 pixels, respectively, and their resulting mean values are listed in Table 3. 


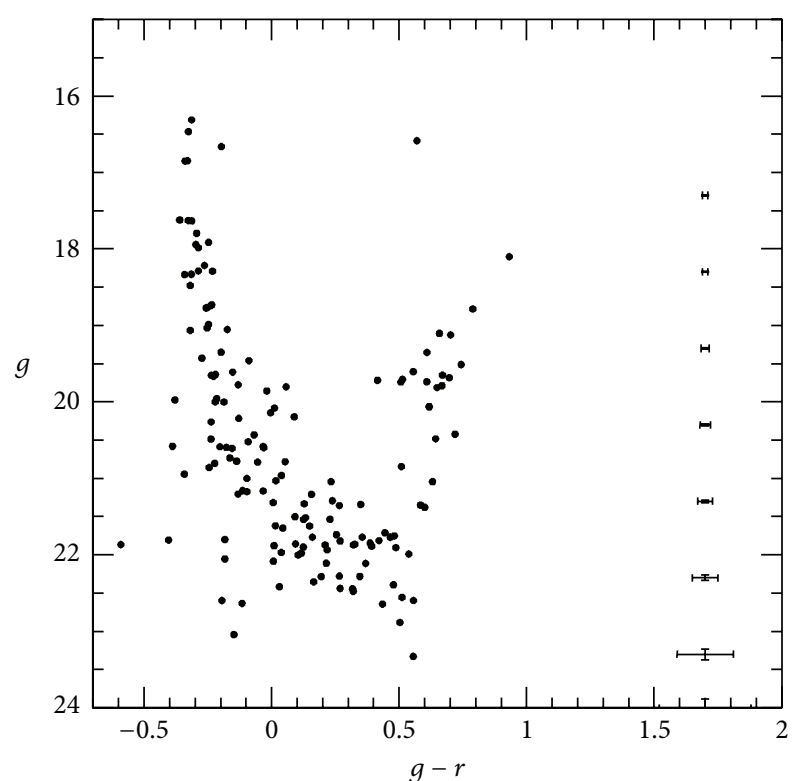

(a)

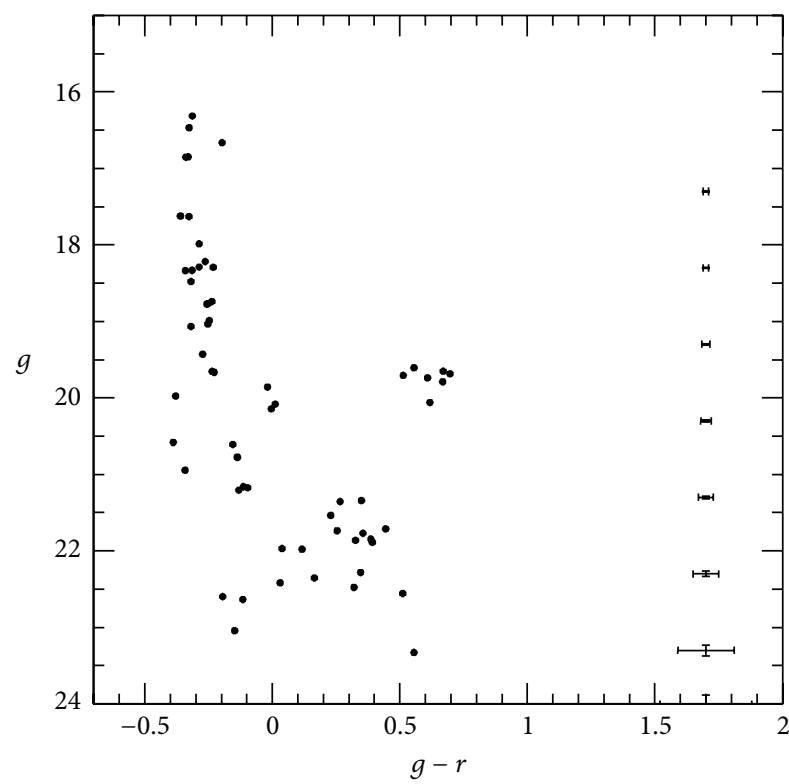

(c)

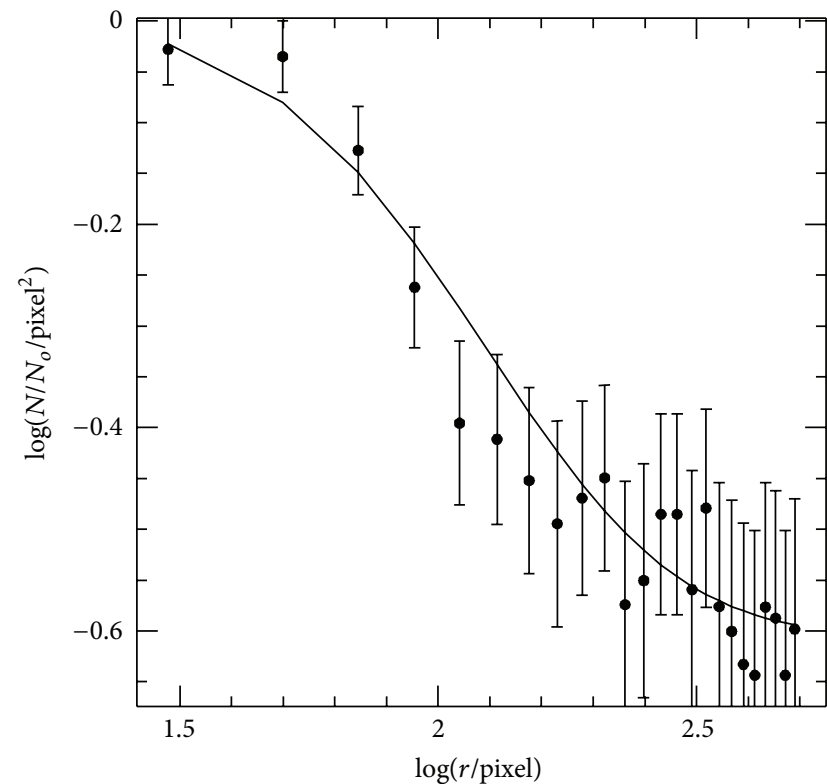

(b)

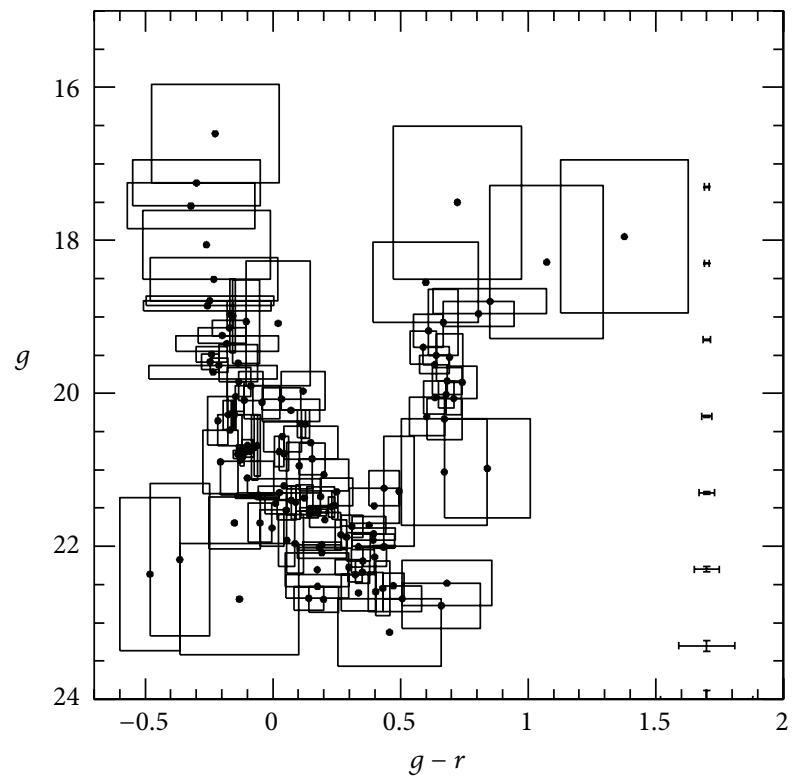

(d)

FIGURE 1: Three extracted CMDs for stars measured in the field of NGC 1768 distributed within the cluster radius (a), the cluster surrounding field for an equal cluster area (d), and the cluster cleaned from field contamination (c). The cluster radial profile is also depicted (b).

TABLE 3: Fundamental parameters for selected LMC star clusters.

\begin{tabular}{|c|c|c|c|c|c|c|c|}
\hline Star clusters & $l(\mathrm{deg})$ & $b(\mathrm{deg})$ & $r_{c}$ (pixel) & $r_{t}($ pixel $)$ & $E(B-V)(\mathrm{mag})$ & $\log (t)$ & $Z$ \\
\hline NGC 1768 & 279.360 & -35.549 & 90 & 800 & 0.05 & $8.00 \pm 0.20$ & 0.008 \\
\hline HS 85 & 278.716 & -35.325 & 160 & 1000 & 0.06 & $8.65 \pm 0.10$ & 0.008 \\
\hline SL 676 & 281.126 & -31.125 & 90 & 500 & 0.07 & $8.80 \pm 0.10$ & 0.008 \\
\hline NGC 2107 & 281.205 & -31.114 & 120 & 1300 & 0.07 & $8.45 \pm 0.10$ & 0.008 \\
\hline NGC 2190 & 285.768 & -29.408 & 200 & 2000 & 0.12 & $9.10 \pm 0.10$ & 0.008 \\
\hline SL 866 & 275.776 & -28.332 & 210 & 2000 & 0.05 & $9.30 \pm 0.10$ & 0.008 \\
\hline
\end{tabular}




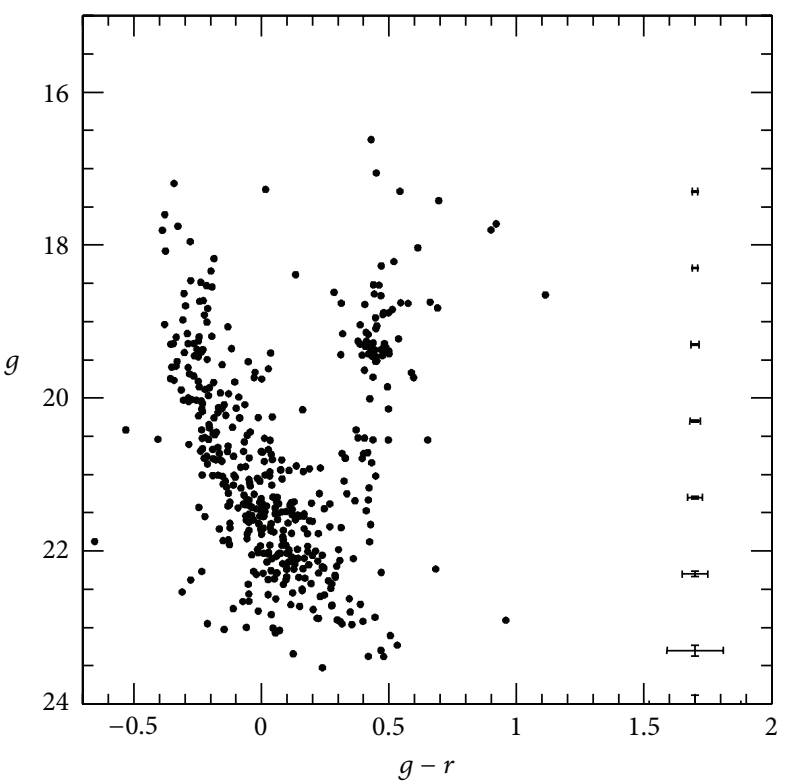

(a)

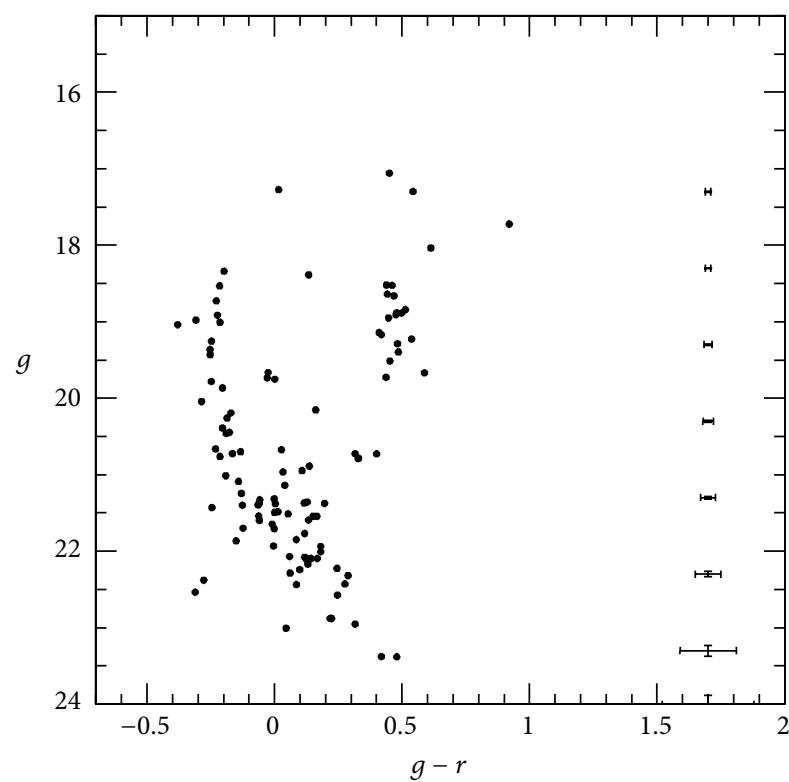

(c)

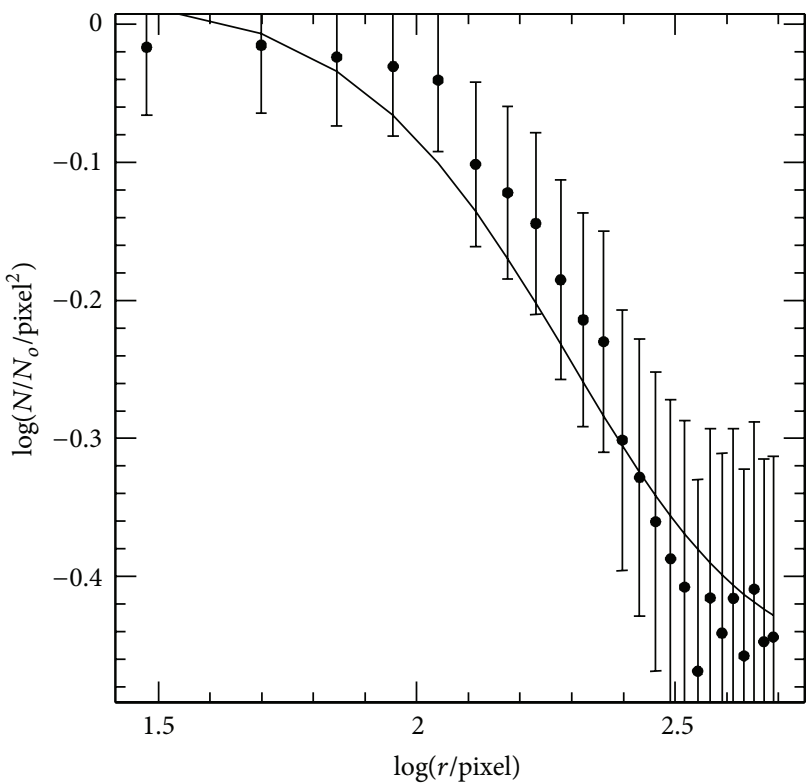

(b)

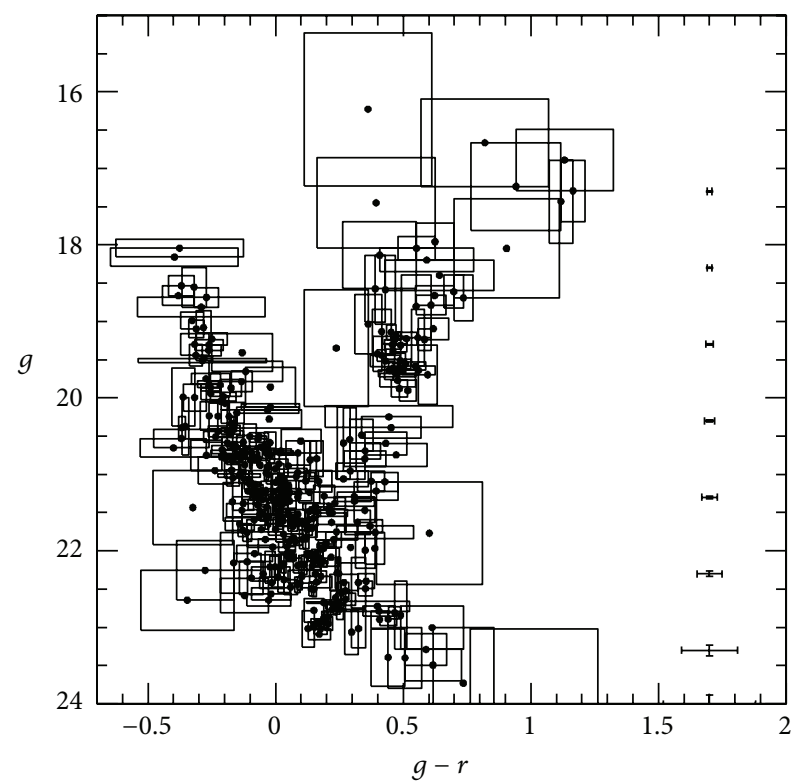

(d)

FIGURE 2: Three extracted CMDs for stars measured in the field of HS 85 distributed within the cluster radius (a), the cluster surrounding field for an equal cluster area (d), and the cluster cleaned from field contamination (c). The cluster radial profile is also depicted (b).

We then constructed three CMDs covering different circular extractions as shown in Figures 1-6 (upper-left, bottom-left, and bottom-right panels). The upper-left panel corresponds to the observed cluster CMD, as built from stars distributed within a circle of radius equal to the cluster radius. The bottom-left panel depicts the resulting cleaned cluster CMD, once the decontamination of field stars was performed, while the bottom-right panel shows a reference field star CMD built from stars distributed within an equal cluster area.
The observational error bars are drawn on the right hand of each panel. As can be seen, the observed cluster CMDs exhibit the most obvious traits main sequences (MSs) which vary in extent and in number of stars, besides the presence of red clump (RC) and red giant branch (RGB) stars. In some cases, populous Sub-Giant Branches are also visible. Note that all these features are also seen in the field star CMDsalthough at a different stellar density level-which reflect the LMC composite stellar populations. The comparison of 


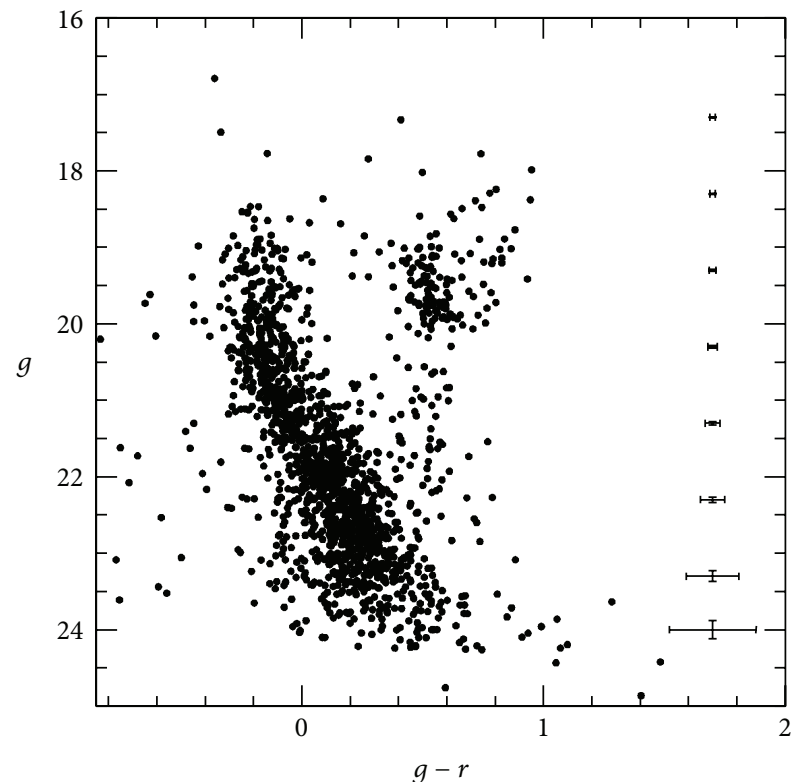

(a)

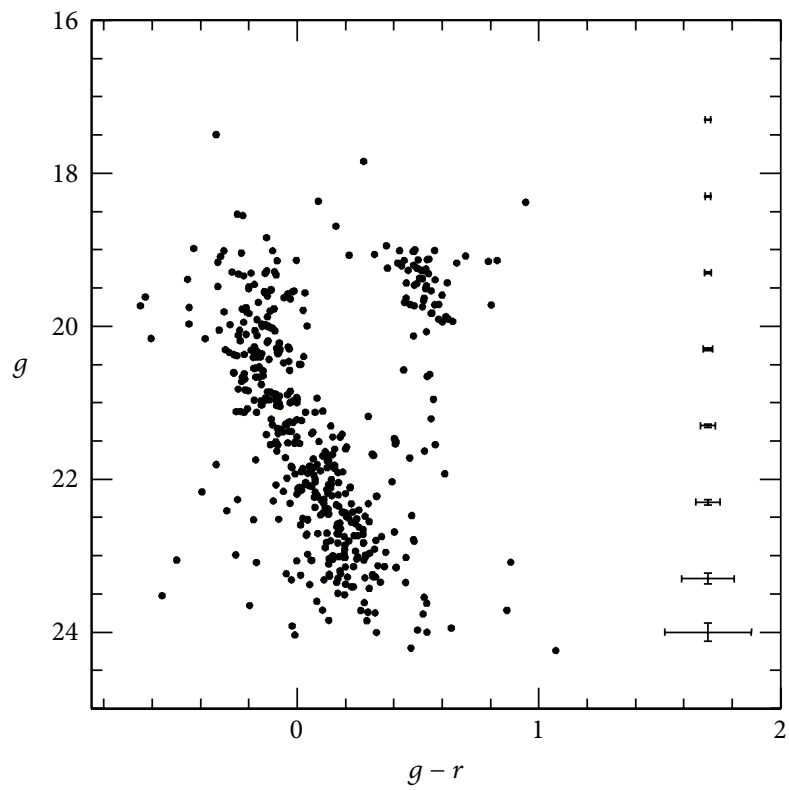

(c)

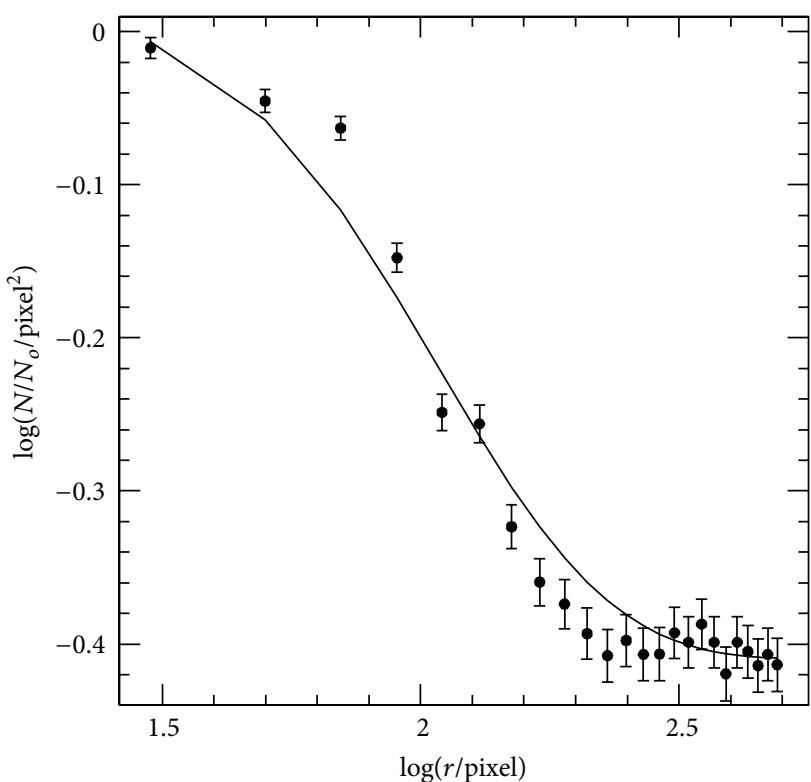

(b)

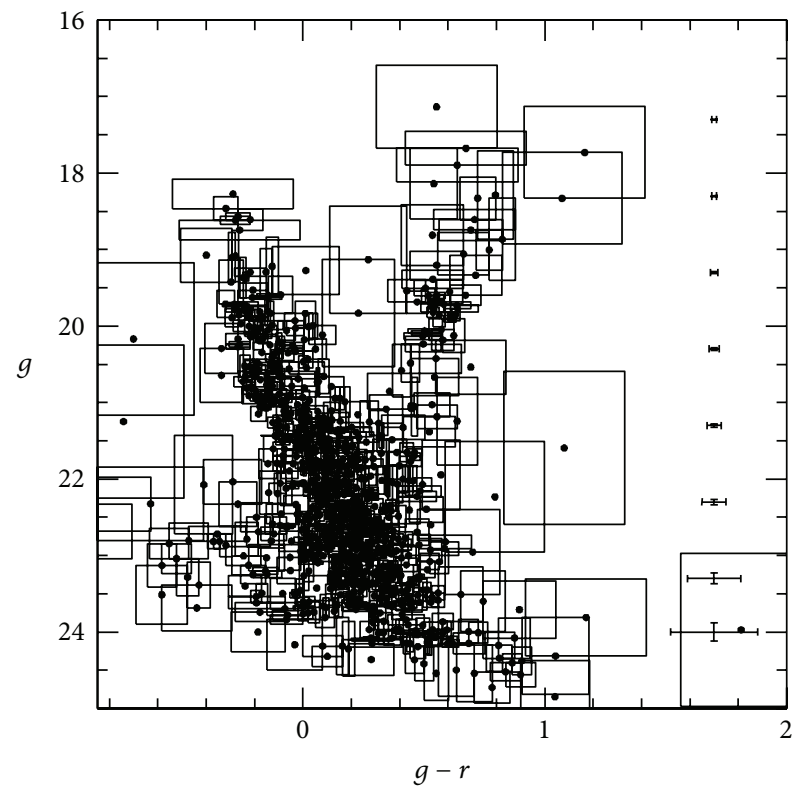

(d)

FIGURE 3: Three extracted CMDs for stars measured in the field of SL 676 distributed within the cluster radius (a), the cluster surrounding field for an equal cluster area (d), and the cluster cleaned from field contamination (c). The cluster radial profile is also depicted (b).

the observed cluster and reference field star CMDs clearly becomes in a robust evidence that field star decontamination is needed in order to disentangle the fiducial cluster features.

Despite the fact that some residual of the field star decontamination is unavoidable, the cleaned cluster CMDs reveal that we are dealing with clusters spread in a relatively wide age range. NGC 1768, HS 85, SL 676, and NGC 2107 appear to be relatively or moderately young star clusters, whereas NGC 2190 and SL 866 seem to be of intermediate age. In addition, SL 676, NGC 2190, and possible NGC 2107 show
RCs with an elongated or secondary structure which resemble that of clusters with evidence of age spread (e.g., [15-17]).

\section{Cluster Fundamental Parameters}

Based on the cleaned cluster CMDs, we followed the common procedure of matching theoretical isochrones in order to find the ones which best reproduce the fiducial cluster features. We chose the evolutionary models developed by 


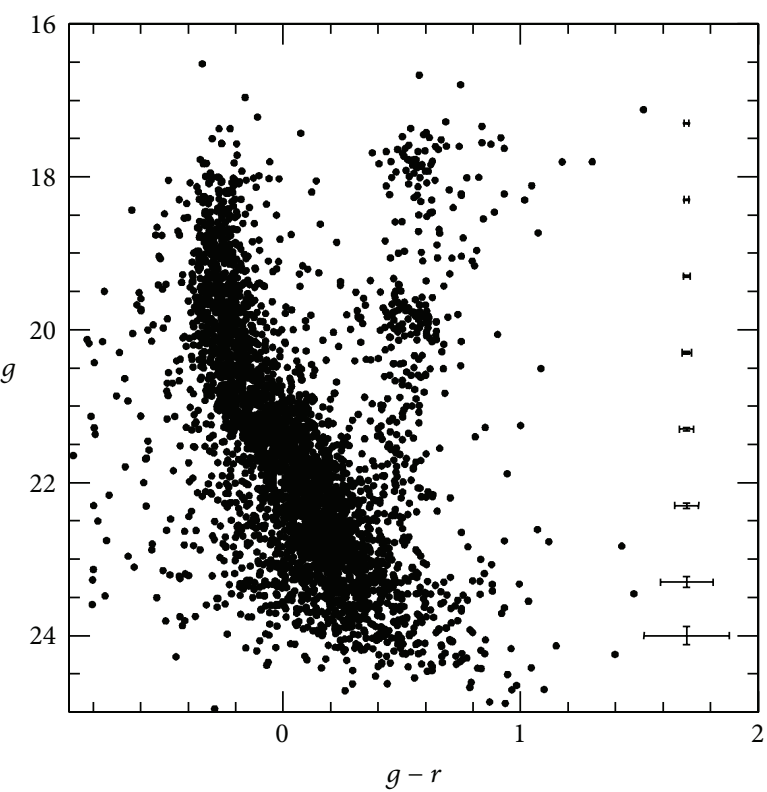

(a)

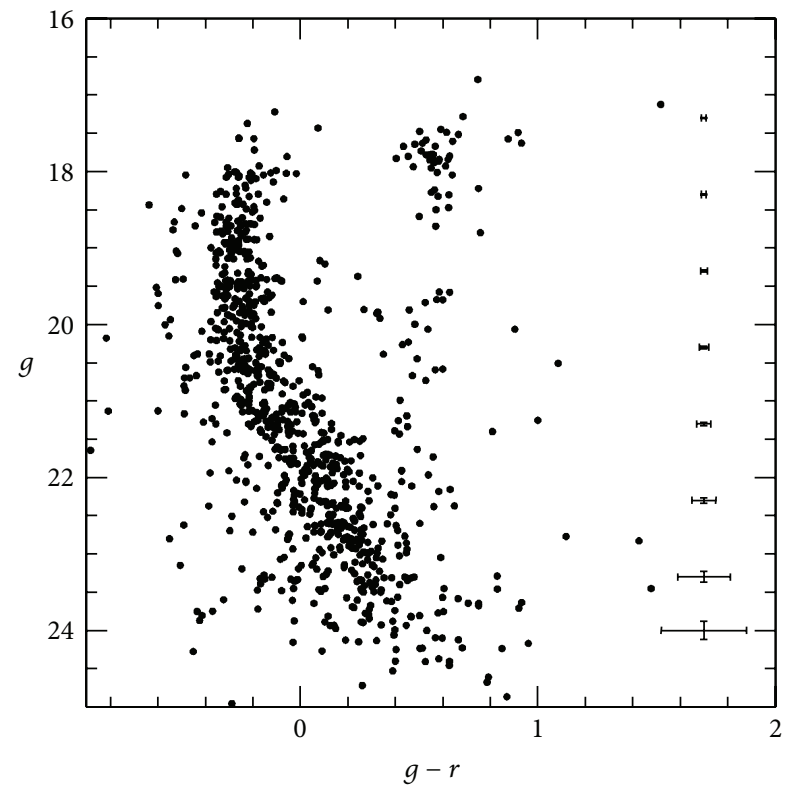

(c)

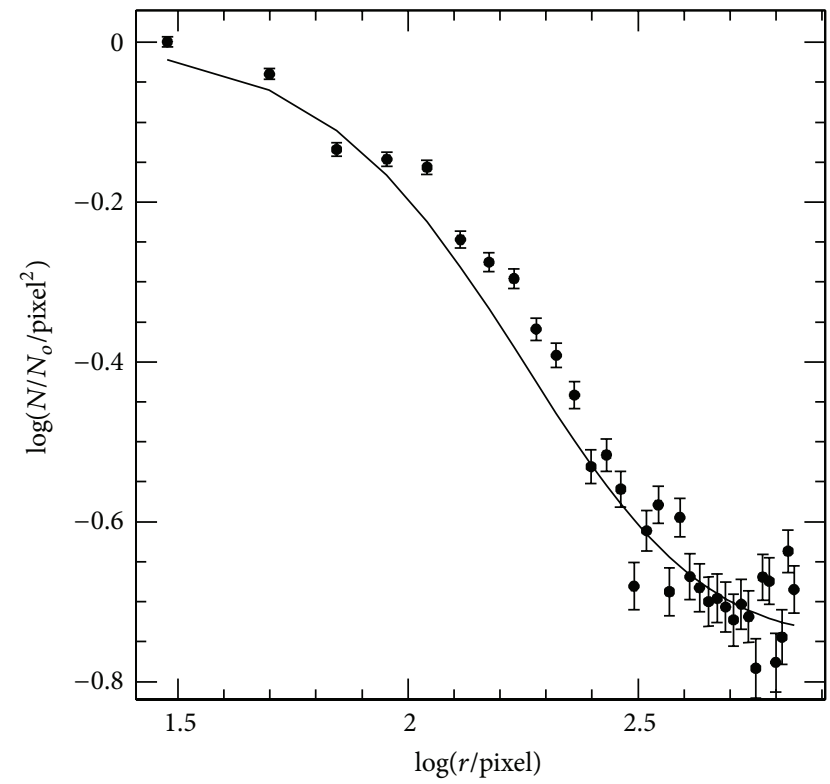

(b)

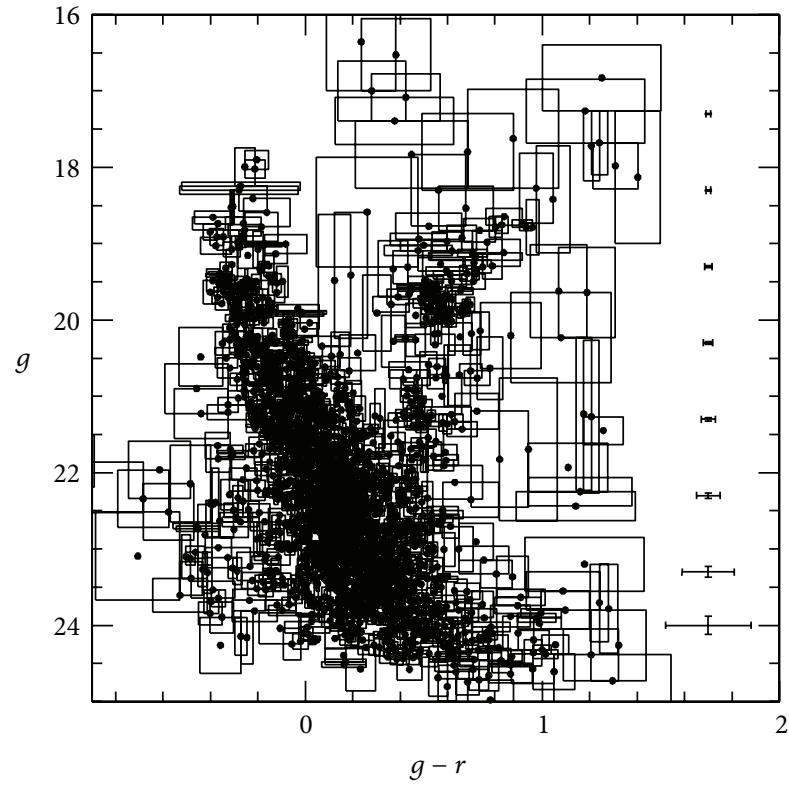

(d)

FIGURE 4: Three extracted CMDs for stars measured in the field of NGC 2107 distributed within the cluster radius (a), the cluster surrounding field for an equal cluster area (d), and the cluster cleaned from field contamination (c). The cluster radial profile is also depicted (b).

Marigo et al. [10] for three different metallicities $Z=0.004$, 0.008 , and $0.020([\mathrm{FeH}]=-0.7,-0.4$, and +0.0 , resp. $)$ to evaluate the metallicity effect in the cluster fundamental parameters. The selected values cover the metallicity range for most of the LMC clusters younger than 4 Gyr [9]. Note that cluster metallicity plays an important role when fitting theoretical isochrones. The distinction is mainly evident for the evolved RC and RGB phases. ZAMSs are often less affected by metallicity effects and can even exhibit imperceptible variations for a specific metallicity range within the expected photometric errors.
Before matching the cluster CMDs with theoretical isochrones, we need to adopt the cluster interstellar extinctions and distance moduli. As for the cluster distance moduli, considering the line-of-sight depth of the galaxy to be approximately $6 \mathrm{kpc}$ [18] and bearing in mind that any cluster of the sample could be placed in front of or behind the main body of the LMC, we concluded that the difference in the cluster apparent distance moduli could be as large as $\Delta\left(V-M_{V}\right) \sim 0.15 \mathrm{mag}$, if a value of $50 \mathrm{kpc}$ is adopted for the mean LMC distance. Since $\Delta\left(V-M_{V}\right)$ resulted smaller than the uncertainties when adjusting the isochrones, 


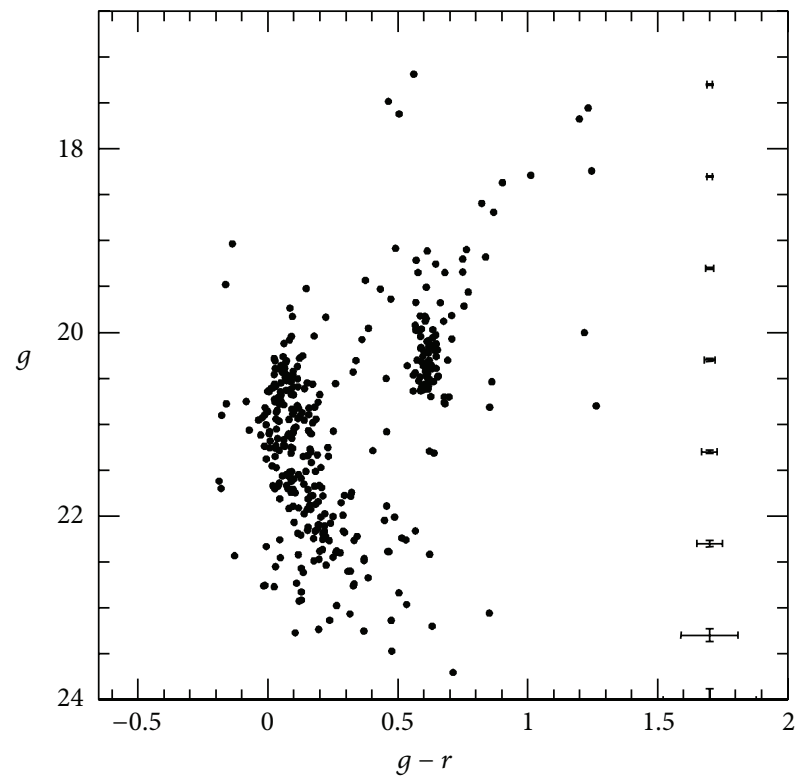

(a)

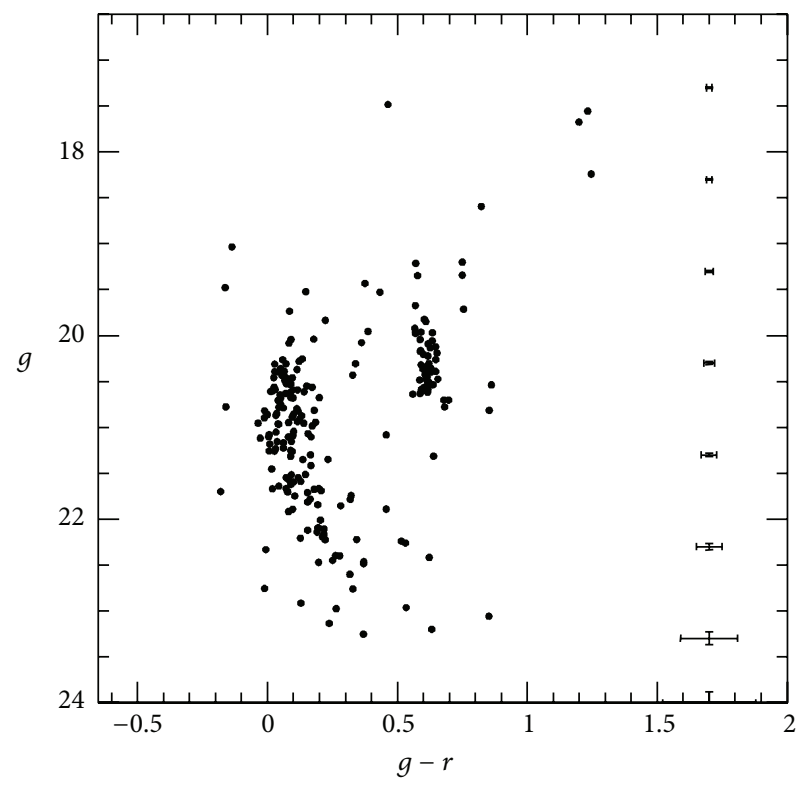

(c)

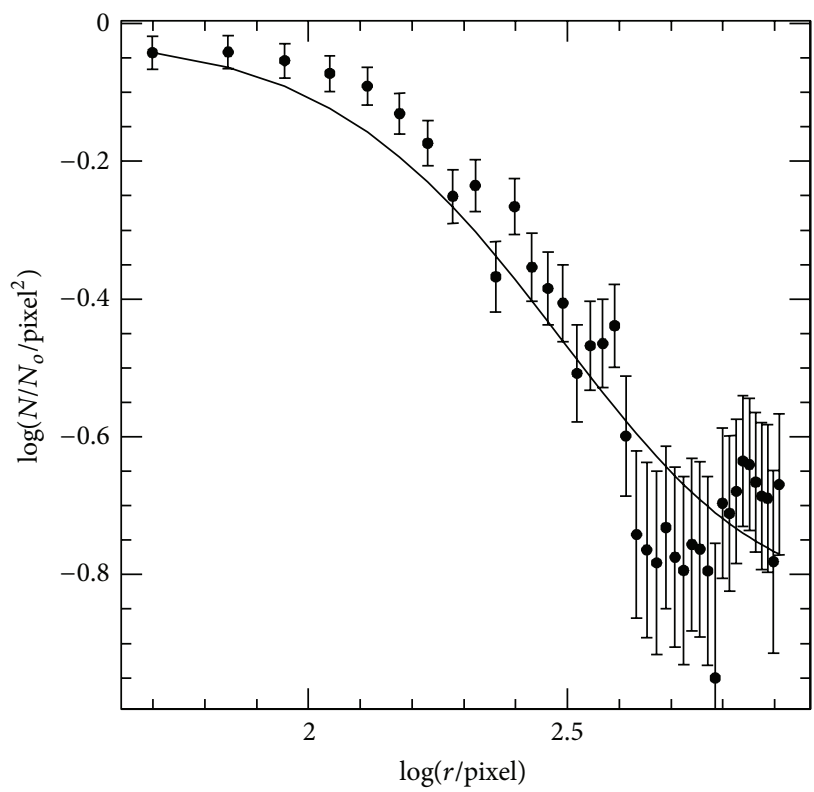

(b)

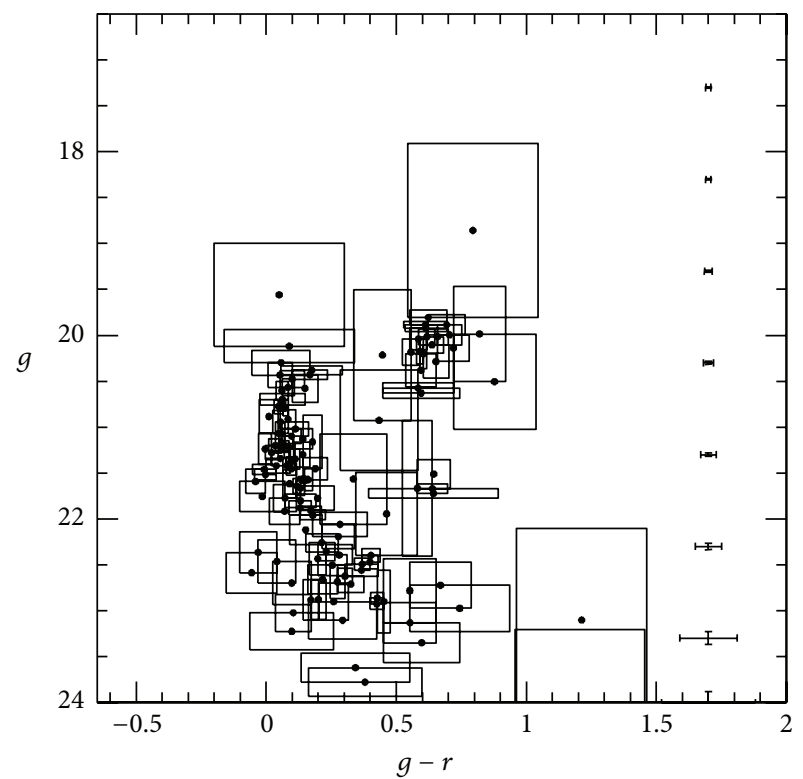

(d)

FIGURE 5: Three extracted CMDs for stars measured in the field of NGC 2190 distributed within the cluster radius (a), the cluster surrounding field for an equal cluster area (d), and the cluster cleaned from field contamination (c). The cluster radial profile is also depicted (b).

the simple assumption of adopting a unique value for the distance modulus for all the clusters should not dominate the error budget in our final results. For this reason, we adopted for all the clusters the value of the LMC distance modulus $(m-M)_{o}=18.50 \pm 0.10$ recently reported by Glatt et al. [3].

The estimation of cluster reddening values was made by interpolating the extinction maps of Burstein and Heiles ([19], hereafter $\mathrm{BH})$. BH maps were obtained from $\mathrm{HI}$ $(21 \mathrm{~cm})$ emission data for the southern sky. They furnish us with foreground $E(B-V)$ color excesses which depend on the Galactic coordinates. We also derived the values of
$E(B-V)$ provided by Haschke et al. ([20], hereafter HGD) based on photometry of RR Lyrae ab stars obtained by the third phase of the Optical Gravitational Lensing Experiment (OGLE III). Although two cluster fields resulted to be outside their extinction maps (NGC 2190 and SL 866), we found a fairly good agreement for the remaining four star clusters of $\Delta\left(E(B-V)_{\mathrm{BH}-\mathrm{HG}}\right)=(-0.026 \pm 0.022) \mathrm{mag}$. We also compared the $E(B-H)_{\mathrm{BH}}$ values with those coming from the Schlegel et al. ([21], hereafter SFD) full-sky $100 \mu \mathrm{m}$ dust emission maps. However, their values deviate for star clusters located in the LMC bar or arms [22] due to saturation of 


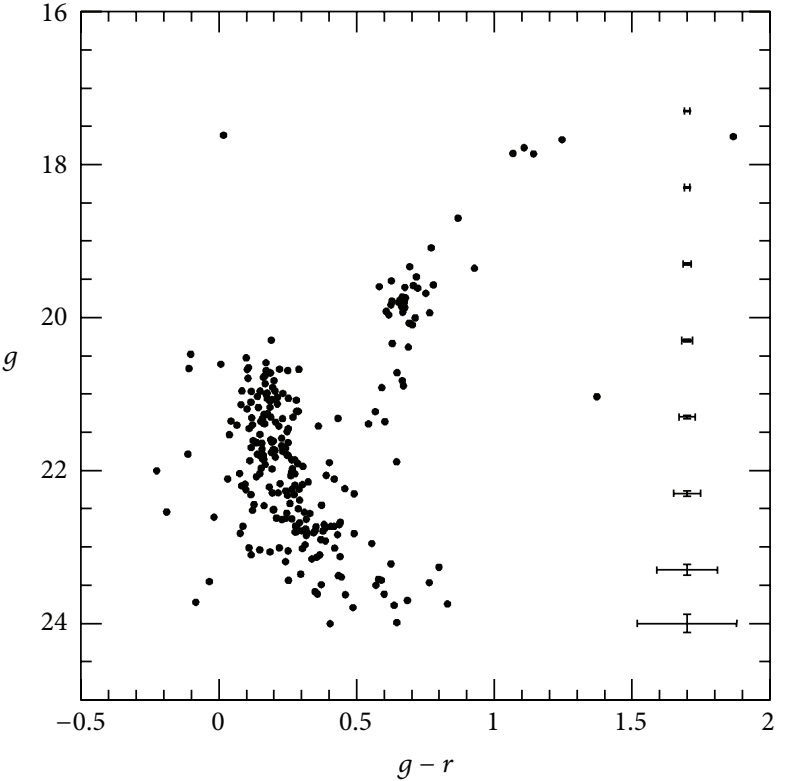

(a)

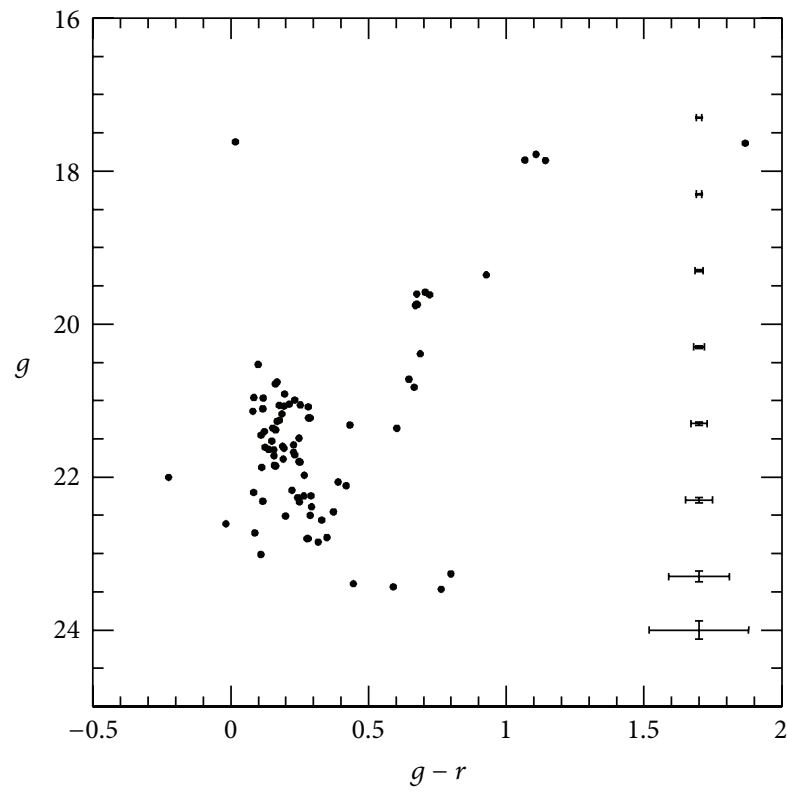

(c)

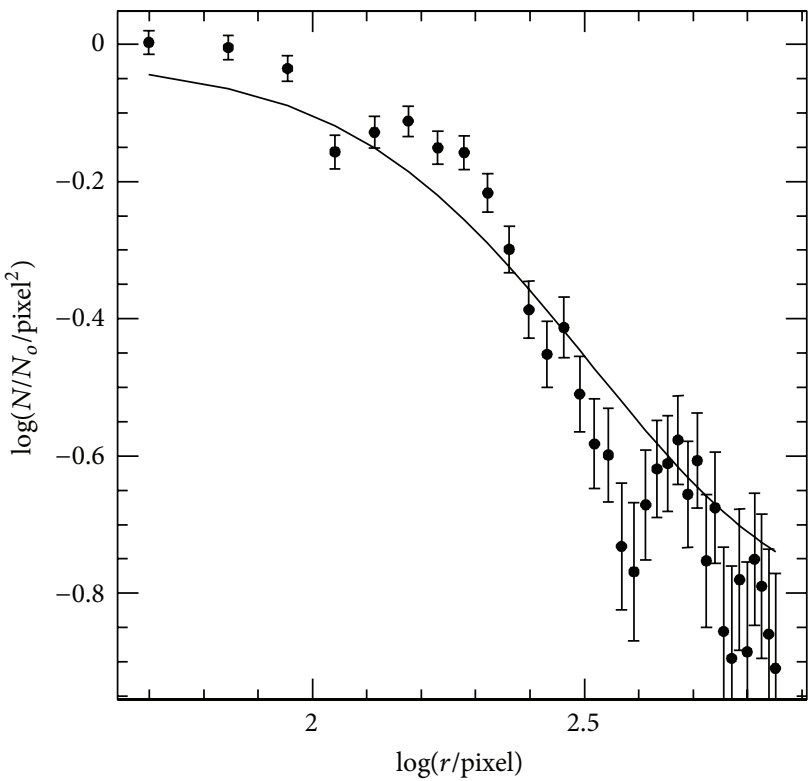

(b)

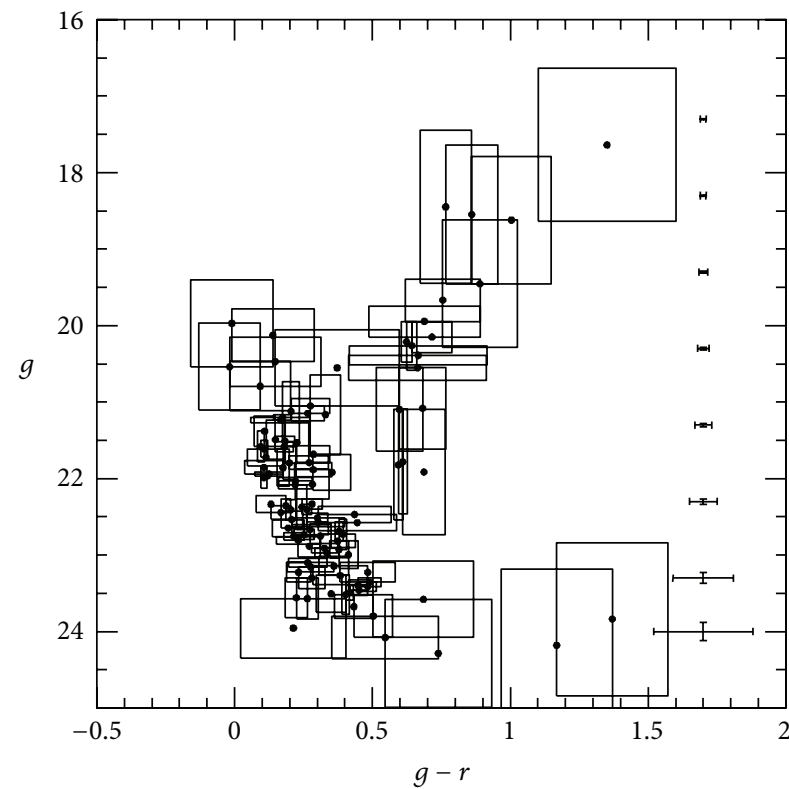

(d)

FIGURE 6: Three extracted CMDs for stars measured in the field of SL 866 distributed within the cluster radius (a), the cluster surrounding field for an equal cluster area (d), and the cluster cleaned from field contamination (c). The cluster radial profile is also depicted (b).

H I emission. This is the case of NGC 1768, located in the northwest end of the bar; HS 85, located in the northwest arm, and SL 676 and NGC 2107 located in the southeast end of the bar, respectively. For NGC 2190 and SL 866, which are placed in the southeastern and northeastern outer disk, respectively, the agreement between $\mathrm{BH}$ and SFD reddenings resulted in satisfactory $\left(\Delta\left(E(B-V)_{\mathrm{BH}-\mathrm{SFD}}\right)=(-0.020 \pm 0.005)\right.$ mag). Table 3 lists the adopted $E(B-V)_{\mathrm{BH}}$ color excesses. We adopted $R=A_{V} / E(B-V)=3.1$ to convert color excess to extinction and used the equations $A_{g} / A_{V}=1.199$ and $A_{r} / A_{V}=0.858$ [23] to evaluate the total extinctions in $A_{g}$ and $A_{r}$. Finally, we used $E(g-r) / A_{V}=0.341$ for the selective extinction in the SDSS system.

We then selected a set of isochrones and superimposed them to the cluster CMDs, once they were properly shifted by the corresponding $E(g-r)$ color excesses and by the LMC distance modulus. In the matching procedure, we used seven different isochrones for each metallicity level, ranging from slightly younger than the derived cluster age to slightly older. Finally, we adopted as the cluster age the one corresponding to the isochrone which best reproduced the cluster main features in the CMD, bearing in mind 

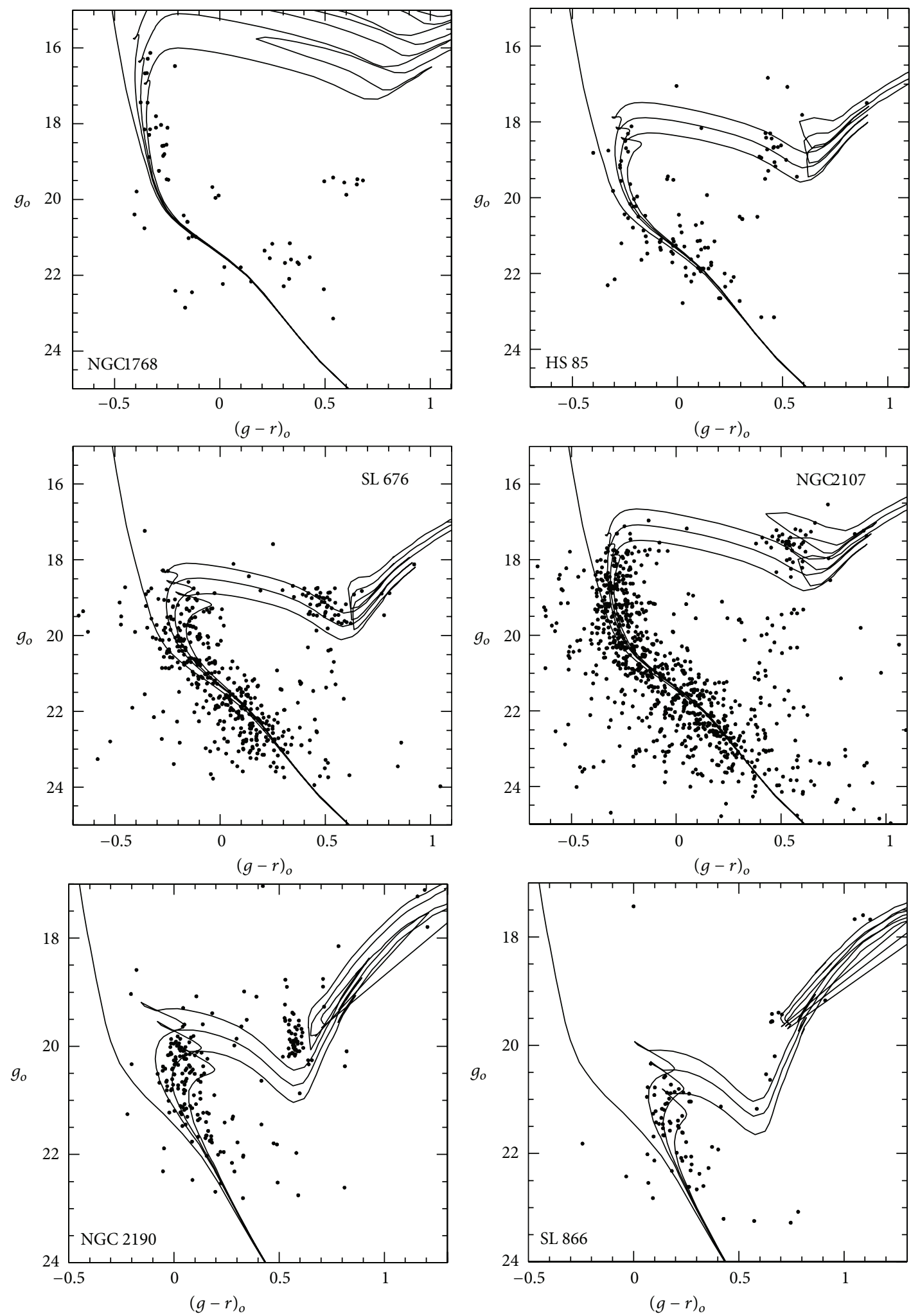

Figure 7: The ZAMS and three isochrones $(Z=0.008)$ from Marigo et al. [10] superimposed to LMC cluster CMDs. The youngest isochrone corresponds to $\log (t)-\sigma(\log (t))$ (see Table 3 ), whereas the isochrone separation is $\Delta(\log (t))=0.10$. 
the observational error bars and the errors in $E(g-r)$ and $(m-M)_{o}$ as well. The presence of RCs and/or RGBs in some cluster CMDs made the fitting procedure easier. We noted, however, that the theoretically computed bluest stage during the He-burning core phase is redder than the observed RC in the CMDs of some clusters, a behaviour already detected in other studies of Galactic and Magellanic Cloud clusters (e.g., [24] and references therein). Notice that we do not provide with metallicity errors, since we only used three prearranged values in the isochrone matching. However, for the sake of the subsequent analysis, the metallicity values adopted are in excellent agreement with those for LMC clusters of similar ages [3]. In Figure 7, we plotted, for each cluster CMD, the isochrone of the adopted cluster age and two additional isochrones bracketing the derived age. The ages of the bracketing isochrones were estimated by taking into account the observed dispersion in the cluster CMDs. The ages of the adopted isochrones and their corresponding metallicities for the cluster sample are listed in Table 3.

\section{Analysis and Discussion}

As far as we are aware from searching the literature, only NGC 1768 has a previous age estimate. Glatt et al. [3] obtained an age of $\log (t)=7.8 \pm 0.4$ in fairly good agreement with our present value, although their uncertainty is noticeably larger. Glatt et al. have used data from the Magellanic Cloud Photometric Surveys [25] to build the cluster CMD. Although they mention that field contamination is a severe effect in the extracted cluster CMDs and therefore influences the age estimates, no decontamination from field CMDs was carried out. Their large age errors could reflect the composite stellar populations of the LMC bar field towards which the cluster is projected.

SL 676 and NGC 2017 resulted to be a cluster pair relatively close in age, with an age difference of (350 \pm 210) Myr. These objects present an angular separation in the sky of $4.1^{\prime}$, which is equivalent to $59.6 \mathrm{pc}$. However, since the upper separation limit for binary LMC star clusters is $\sim 20 \mathrm{pc}$ $[26,27]$, we concluded that they do not constitute a physical system.

Finally, NGC 2190 and SL 866 resulted to be intermediateage star clusters. According to their positions in the galaxy, the resulting ages are in good agreement with those of star clusters placed at a similar deprojected distance from the LMC center, whereas the present metallicities more metalrich [6]. Comparing the cluster ages and metallicities with those of their respective surrounding star fields [3], we found that the latter are older $(\langle t\rangle \sim 5 \mathrm{Gyr})$ and more metalpoor $([\mathrm{Fe} / \mathrm{H}] \sim-1.0 \mathrm{dex})$. The remarkable different ages and metallicities of the star clusters and the dominant field stellar populations could be explained if we assume that the clusters were born in other parts of the galaxy and, because of their orbital motions, they are observed at the current locations. Notice that the ages of NGC 2190 and SS 866 are encompassed within the well-known star cluster bursting formation epoch [8], so that they could have been formed in regions where the cluster burst took place.

\section{Summary}

In this study, we present for the first time CCD SDSS gr photometry of stars in the field of poorly studied LMC star clusters, namely, NGC 1768, HS 85, SL 676, NGC 2107, NGC 2190, and SL 866. The star clusters are spread throughout the bar, arms, and outer disk of the galaxy. The data were obtained at the Gemini South telescope with the GMOS attached. We are confident that the photometric data yield accurate morphology and position of the main cluster features in the CMDs. We applied a subtraction procedure to statistically clean the cluster CMDs from field star contamination in order to disentangle cluster features from those belonging to their surrounding fields. The technique makes use of variable cells in order to reproduce the field CMD as closely as possible. We trace their stellar density radial profiles from star counts performed over the cleaned field star datasets. From the density profiles, we adopted cluster radii defined as the distance from the cluster center where the stellar density profile intersects with the background level and derived the radii at the FWHM of the radial profile. We then built CMDs with cluster features being clearly identified. Using the cleaned cluster CMDs, we estimated ages and metallicities from matching theoretical isochrones computed for the SDSS system. When adjusting a subset of isochrones, we took into account the LMC distance modulus and the individual star cluster color excesses. The studied star clusters turned out to cover a relatively wide age range, from relatively young up to intermediate-age clusters. We found that SL 676 and NGC 2107 are not binary clusters but aligned along the same line of sight, while NGC 2109 and SL 866 are intermediate-age and slightly metal-poor clusters located in the outer disk where the dominant stellar populations are older and more metalpoor. The remarkably different ages and metallicities could be explained if we consider the star cluster orbital motions.

\section{Conflict of Interests}

The author declares that there is no conflict of interests regarding the publication of this paper.

\section{Acknowledgments}

This work was partially supported by the Argentinian institutions CONICET and Agencia Nacional de Promoción Cientifica y Tecnológica (ANPCyT). This paper was prepared with the AAS LATEX macros v5.2.

\section{References}

[1] E. Bica, C. Bonatto, C. M. Dutra, and J. F. C. Santos Jr., "A general catalogue of extended objects in the Magellanic system," Monthly Notices of the Royal Astronomical Society, vol. 389, no. 2, pp. 678-690, 2008.

[2] E. Chiosi, A. Vallenari, E. V. Held, L. Rizzi, and A. Moretti, "Age distribution of young clusters and field stars in the Small Magellanic Cloud," Astronomy and Astrophysics, vol. 452, no. 1, pp. 179-193, 2006. 
[3] K. Glatt, E. K. Grebel, and A. Koch, "Ages and luminosities of young SMC/LMC star clusters and the recent star formation history of the clouds," Astronomy and Astrophysics, vol. 517, no. 7,2010.

[4] A. E. Piatti, D. Geisler, E. Bica et al., "A new giant branch clump structure in the Large Magellanic Cloud," Astronomical Journal, vol. 118, no. 6, pp. 2865-2874, 1999.

[5] A. E. Piatti, A. Sarajedini, D. Geisler, E. Bica, and J. J. Clariá, "Constraining the LMC cluster age gap: washington photometry of NGC 2155 and SL 896 (LW 480)," Monthly Notices of the Royal Astronomical Society, vol. 329, no. 3, pp. 556-566, 2002.

[6] A. E. Piatti, D. Geisler, A. Sarajedini, and C. Gallart, "Washington photometry of five star clusters in the Large Magellanic Cloud," Astronomy and Astrophysics, vol. 501, no. 2, pp. 585-593, 2009.

[7] A. E. Piatti, J. J. Clariá, M. C. Parisi, and A. V. Ahumada, "First estimates of the fundamental parameters of three large Magellanic Cloud clusters," Publications of the Astronomical Society of the Pacific, vol. 123, no. 903, pp. 519-530, 2011.

[8] A. E. Piatti, "New insights on the bursting formation of star clusters in the Large Magellanic Cloud," Monthly Notices of the Royal Astronomical Society, vol. 418, no. 1, pp. L40-L44, 2011.

[9] A. E. Piatti and D. Geisler, "The age-metallicity relationship of the large Magellanic Cloud field star population from widefield washington photometryextended star formation in the intermediate-age large Magellanic Cloud star cluster ngc 2209," Astronomical Journal, vol. 145, no. 1, p. 17, 2013.

[10] P. Marigo, L. Girardi, A. Bressan, M. A. T. Groenewegen, L. Silva, and G. L. Granato, "Evolution of asymptotic giant branch stars: II. Optical to far-infrared isochrones with improved TP-AGB models," Astronomy and Astrophysics, vol. 482, no. 3, pp. 883905, 2008.

[11] M. Fukugita, T. Ichikawa, J. E. Gunn, M. Doi, K. Shimasaku, and D. P. Schneider, "The sloan Digital Sky Survey photometric system," Astronomical Journal, vol. 111, no. 4, pp. 1748-1756, 1996.

[12] P. B. Stetson, L. E. Davis, and D. R. Crabtree, "Future development of the DAOPHOT crowded-field photometry package," in CCDs in Astronomy, G. H. Jacoby, Ed., vol. 8, p. 289, Astronomical Society of the Pacific Conference Series, San Francisco, Calif, USA, 1990.

[13] A. E. Piatti and E. Bica, "Washington photometry of candidate star clusters in the Small Magellanic Cloud," Monthly Notices of the Royal Astronomical Society, vol. 425, no. 4, pp. 3085-3093, 2012.

[14] I. King, "The structure of star clusters. I. an empirical density law ," Astronomical Journal, vol. 67, p. 471, 1962.

[15] A. P. Milone, L. R. Bedin, G. Piotto, and J. Anderson, "Multiple stellar populations in Magellanic Cloud clusters," Astronomy and Astrophysics, vol. 497, no. 3, pp. 755-771, 2009.

[16] S. C. Keller, A. D. Mackey, and G. S. Da Costa, "Extended star formation in the intermediate-age large magellanic cloud star cluster Ngc 2209," The Astrophysical Journal, vol. 761, no. 1, article L5, 2012.

[17] A. E. Piatti, "A new extended main-sequence turnoff star cluster in the Large Magellanic Cloud," Monthly Notices of the Royal Astronomical Society, vol. 430, no. 3, pp. 2358-2362, 2013.

[18] H. H. Crowl, A. Sarajedini, A. E. Piatti et al., "The line-ofsight depth of populous clusters in the Small Magellanic Cloud," Astronomical Journal, vol. 122, no. 1, pp. 220-231, 2001.
[19] D. Burstein and C. Heiles, "Reddenings derived from H I and galaxy counts-Accuracy and maps," Astronomical Journal, vol. 87, pp. 1165-1189, 1982.

[20] R. Haschke, E. K. Grebel, and S. Duffau, "New optical reddening maps of the large and small magellanic clouds," Astronomical Journal, vol. 141, no. 5, article 158, 2011.

[21] D. J. Schlegel, D. P. Finkbeiner, and M. Davis, "Maps of dust infrared emission for use in estimation of reddening and cosmic microwave background radiation foregrounds," Astrophysical Journal Letters, vol. 500, no. 2, pp. 525-553, 1998.

[22] J. Harris and D. Zaritsky, "The star formation history of the large magellanic cloud," Astronomical Journal, vol. 138, no. 5, pp. 1243-1260, 2009.

[23] X. Fan, "Simulation of stellar objects in SDSS color space," Astronomical Journal, vol. 117, no. 5, pp. 2528-2551, 1999.

[24] A. E. Piatti, J. J. Clariá, E. Bica, D. Geisler, A. V. Ahumada, and L. Girardi, "Washington photometry of 14 intermediate-age to old star clusters in the Small Magellanic Cloud," Monthly Notices of the Royal Astronomical Society, vol. 417, no. 2, pp. 1559-1575, 2011.

[25] D. Zaritsky, J. Harris, I. B. Thompson, E. K. Grebel, and P. Massey, "The magellanic clouds photomtric survey: the small magellanic cloud stellar catalog and extinction map," Astronomical Journal, vol. 123, no. 2, pp. 855-872, 2002.

[26] R. K. Bathia, M. A. Read, D. Hatzidimitriou, and S. Tritton, "A catalogue of binary star cluster candidates in the Large Magellanic Cloud," Astronomy and Astrophysics Supplement Series, vol. 87, no. 2, pp. 335-352, 1991.

[27] A. Dieball, H. Müller, and E. K. Grebel, "A statistical study of binary and multiple clusters in the LMC", Astronomy and Astrophysics, vol. 391, no. 2, pp. 547-564, 2002. 

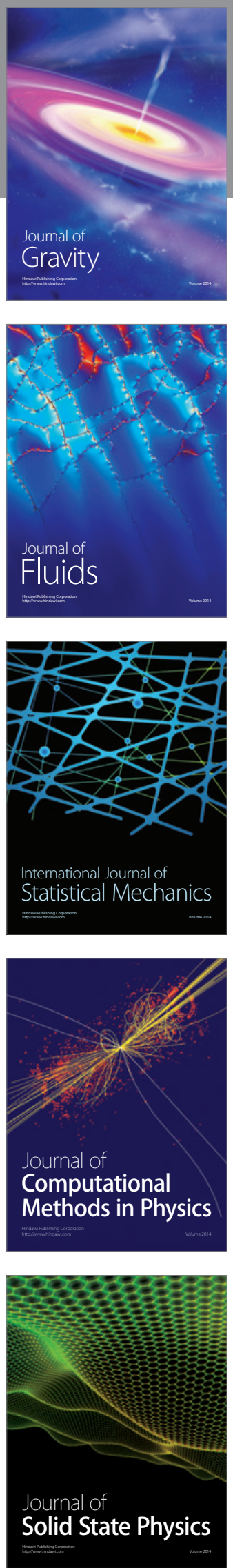

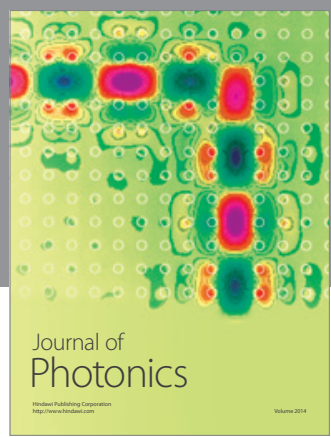

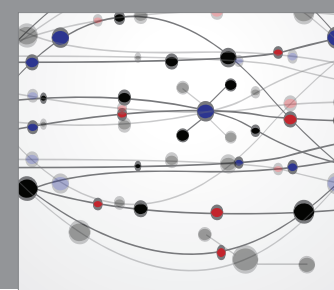

The Scientific World Journal

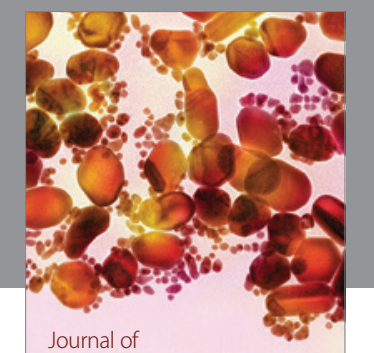

Soft Matter
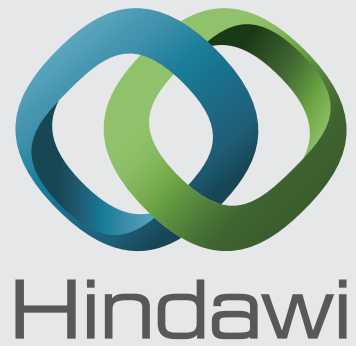

Submit your manuscripts at

http://www.hindawi.com
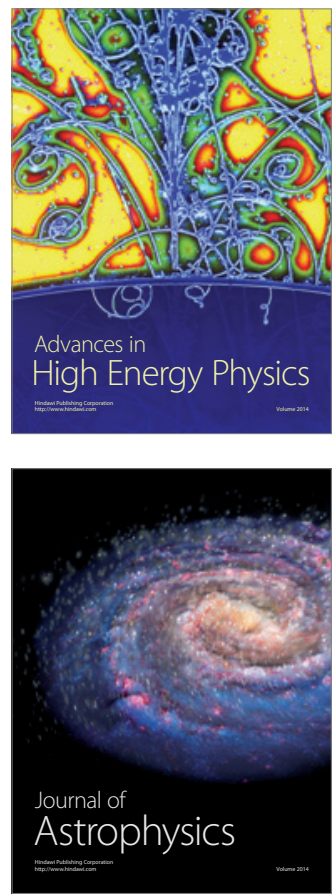
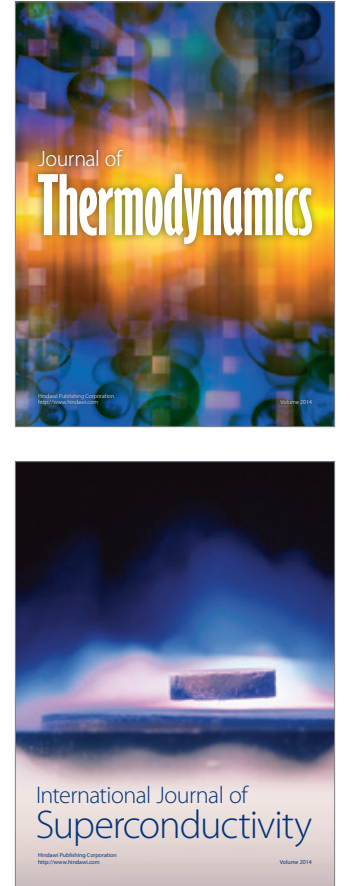
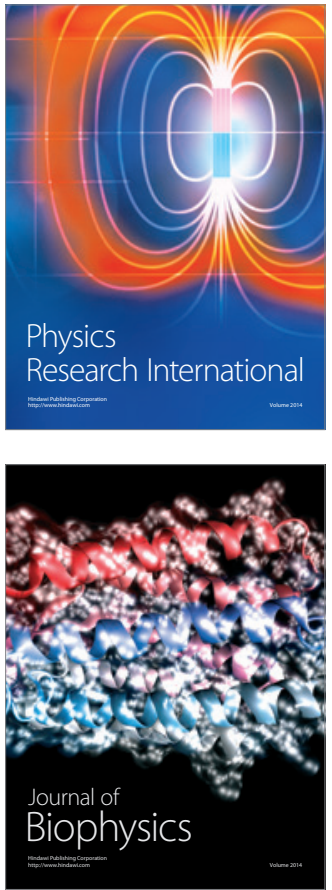
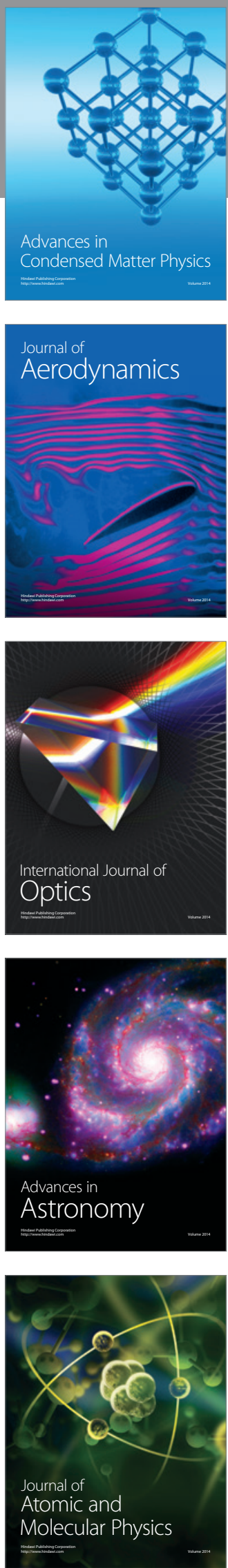